\title{
NGC6334I - Tracing the Gas Motion During A Contemporaneous Maser Flare Event
}

\author{
James O. Chibueze \# \\ South African Radio Astronomy Observatory, Times Media Building, 2 Fir Street, Black River \\ Park, Observatory 7925, South Africa. \\ Centre for Space Research, Physics Department, North-West University, Potchefstroom, 2520, \\ South Africa \\ Department of Physics and Astronomy, University of Nigeria, Carver Building, 1 University \\ Road, Nsukka, 410001, Nigeria \\ E-mail: james.chibueze@gmail.com
}

\section{Tomoya Hirota}

National Astronomical Observatory of Japan, Osawa 2-21-1, Mitaka, Tokyo 181-8588, Japan

\section{Gordon C. MacLeod}

Hartebeesthoek Radio Astronomy Observatory, P.O. Box 443, Krugersdorp 1740, South Africa

\section{Crystal L. Brogan}

NRAO, 520 Edgemont Road, Charlottesville, VA 22903, USA

\section{Todd Hunter}

NRAO, 520 Edgemont Road, Charlottesville, VA 22903, USA

\section{Ruby van Rooyen}

South African Radio Astronomy Observatory, Times Media Building, 2 Fir Street, Black River Park, Observatory 7925, South Africa.

\begin{abstract}
NGC 6334 I showed evidence of episodic accretion event observed as f ares in multiple maser species (water, methanol and hydroxyl) and a brightening of the dust continuum emission in millimetre wavelength. Our multi-epoch water maser observations overlapped with this episodic event. Evidence of compact outf ows and turbulent gas motion can me seen in the region. MM1 (a dominant millimetre source in the region) recently excited $6.7 \mathrm{GHz}$ methanol masers, and the gas motion around it trace the earliest activities associated formation of the driving source(s) in the region.
\end{abstract}

14th European VLBI Network Symposium \& Users Meeting (EVN 2018)

8-11 October 2018

Granada, Spain

\footnotetext{
${ }^{*}$ Speaker.

${ }^{\dagger} \mathrm{JOC}$.
} 


\section{Introduction}

Outburst or flare in maser activities around star-forming regions provide intriguing clues into the ongoing star formation activities in the region. Flares have been observed in a number of sources like Orion KL (Abraham et al. 1981; Omodaka et al. 1999; Hirota et al. 2014), W49N (Liljeström \& Gwinn 2000; Honma et al. 2004), G25.65+1.05 (Lekht et al. 2017; Volvach et al. 2017) and NGC 6334 I (MacLeod et al. 2018; Hunter et al. 2017, 2018; Brogan et al. 2018).

The maser flares have been associated with protostellar accretion outbursts (Hunter et al. 2017; Moscadelli et al. 2017). Hunter et al. (2017) suggested that the accretion outbursts could be the mass-gain mechanism of massive stars, possibly triggered by the presence of a clump of gas close the central core/disk system.

NGC 6334 I (located $~ 1.3 \mathrm{kpc}$; Chibueze et al. 2014) has been identified as a proto-cluster housing high-mass protostars at various evolutionary stages (Hunter et al. 2006, Brogan et al. 2016). MacLeod et al. (2018) reported a flare in multiple maser species including the water $\left(\mathrm{H}_{2} \mathrm{O}\right)$ masers, which simultaneously occurred with the brightening of MM1 dust continuum emission reported by Hunter et al. (2017) based on their Atacama Large Millimetre/sub-millimetre Array (ALMA) observations.

We report follow up very long baseline interferometric (VLBI) observations of the $\mathrm{H}_{2} \mathrm{O}$ masers during the period of the flare.

\section{Observations and Data Reduction}

During the flare period of the $\mathrm{H}_{2} \mathrm{O}$ masers of NGC 6334 I, we conducted a 3-epoch target of opportunity (ToO) observations with a combination of the Korean VLBI Network (KVN) and the VLBI Exploration of Radio Astrometry (VERA), known as KaVA at $22.235080 \mathrm{GHz}$ the rest frequency of the $\mathrm{H}_{2} \mathrm{O}\left(6_{12}-5_{23}\right)$ maser transition. The observations took place on 21 November, 2015 (R15325), 15 December 2015 (R15349) and 4 January 2016, corresponding to a separation of 24 days between the first and second epochs and 20 days between the second and third epochs.

Left-hand circular polarization was received and sampled with a 2-bit quantization. At the VERA stations, a single-beam mode was used in KaVA observations. All the data were recorded at $1 \mathrm{Gbps}(16 \mathrm{MHz} \times 16$ sub-bands) and correlated by the Korea-Japan Joint VLBI Correlator (KJJVC) in Daejeon.

The KaVA data reduction was carried out using the Astronomical Image Processing System (AIPS) software, developed by the National Radio Astronomy Observatory (NRAO). Standard calibration, self-calibration, and imaging procedures were used.

\section{Results}

\section{1 $\mathrm{H}_{2} \mathrm{O}$ maser distribution}

After careful identification of real maser features (devoid of side-lobes or false detections), we obtained the maser map shown in Figure 1. The maser features have local standard of rest (LSR) velocity range of $-62 \mathrm{~km} \mathrm{~s}^{-1}$ to $14 \mathrm{~km} \mathrm{~s}^{-1}$. For easy identification, we have named the maser clusters, cluster $\mathrm{A}-\mathrm{E}$, with cluster $\mathrm{A}$ being the north-most cluster and $\mathrm{E}$ being the south-most 
cluster. The most blue-shifted masers are located in the B cluster, while the most red-shifted ones are in the C cluster. Our maser distribution is consistent with that reported by Brogan et al. (2018) using the JVLA.

Maser cluster A show clear arc-structure (also reported in Brogan et al. 2018) seen around CM2 object. Clear displacements can be seen in the relative positions of the some the maser features as shown in the zoom-in panels of Figure 1.

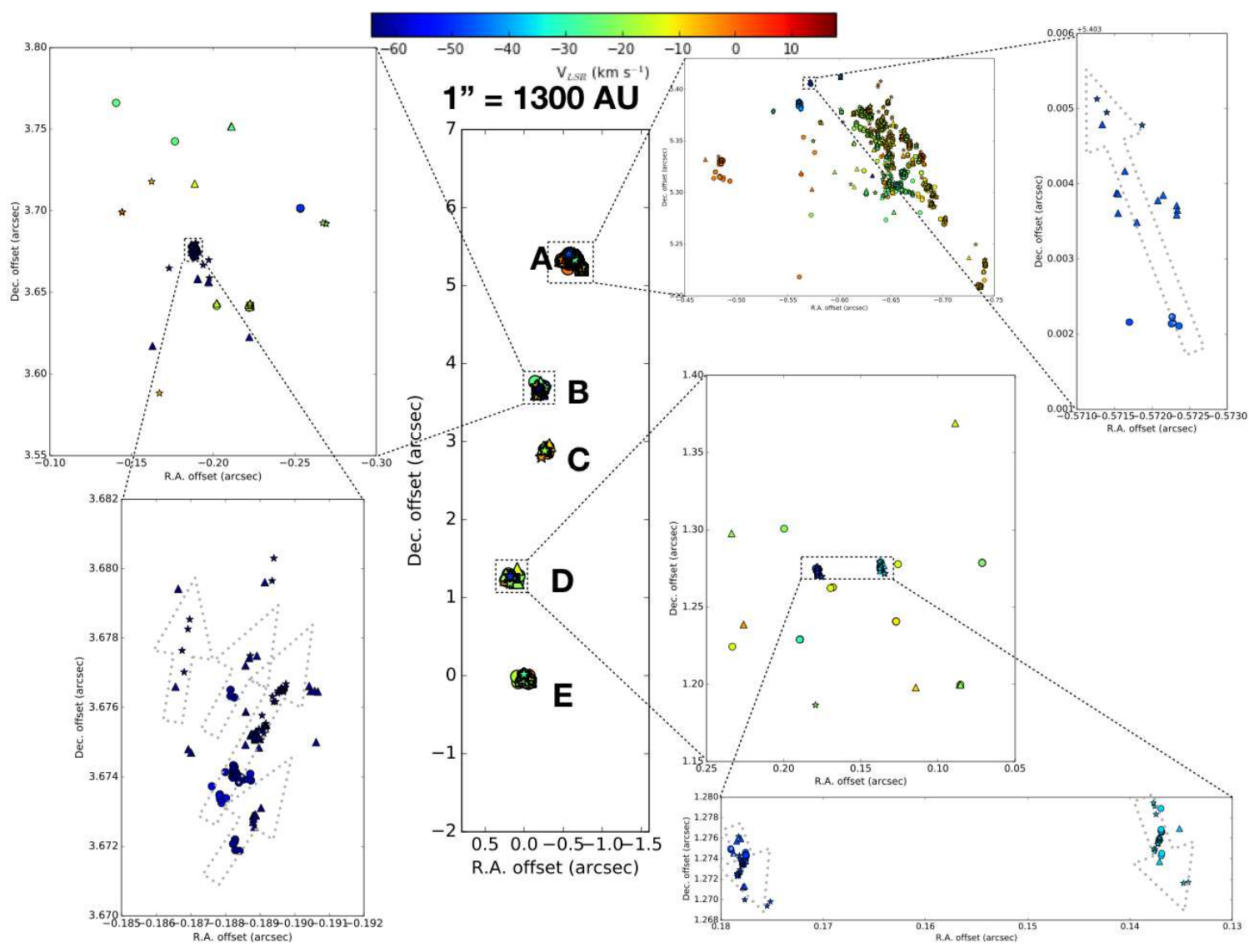

Figure 1: Distribution of the maser features detected in our observations. The filled circles, triangles, and stars represent masers detected in epochs 1, 2, and 3, respectively. The color codes represent the LSR velocities of the maser features.

\section{2 $\mathrm{H}_{2} \mathrm{O}$ maser relative proper motion}

The maser map registration was done with reference maser spot located at the map origin. The reference position was derived from the average of the offsets of the reference maser position in the maps of the 3 epochs. This is to compensate for the effect of the internal motion of the reference maser spot to the relative proper motion of other maser features. We identified masers detected in adjacent epochs and traced the relative proper motion of the maser features. The average proper motion of the masers is $\sim 5 \mathrm{~km} \mathrm{~s}^{-1}$.

We obtained ten dozens of the proper motion vectors for the maser features associated with maser groups A - E (see Figure 2. Overall gas motion traced by the maser kinematics is consistent with the north-south bipolar outflow reported by Brogan et al. (2018). 

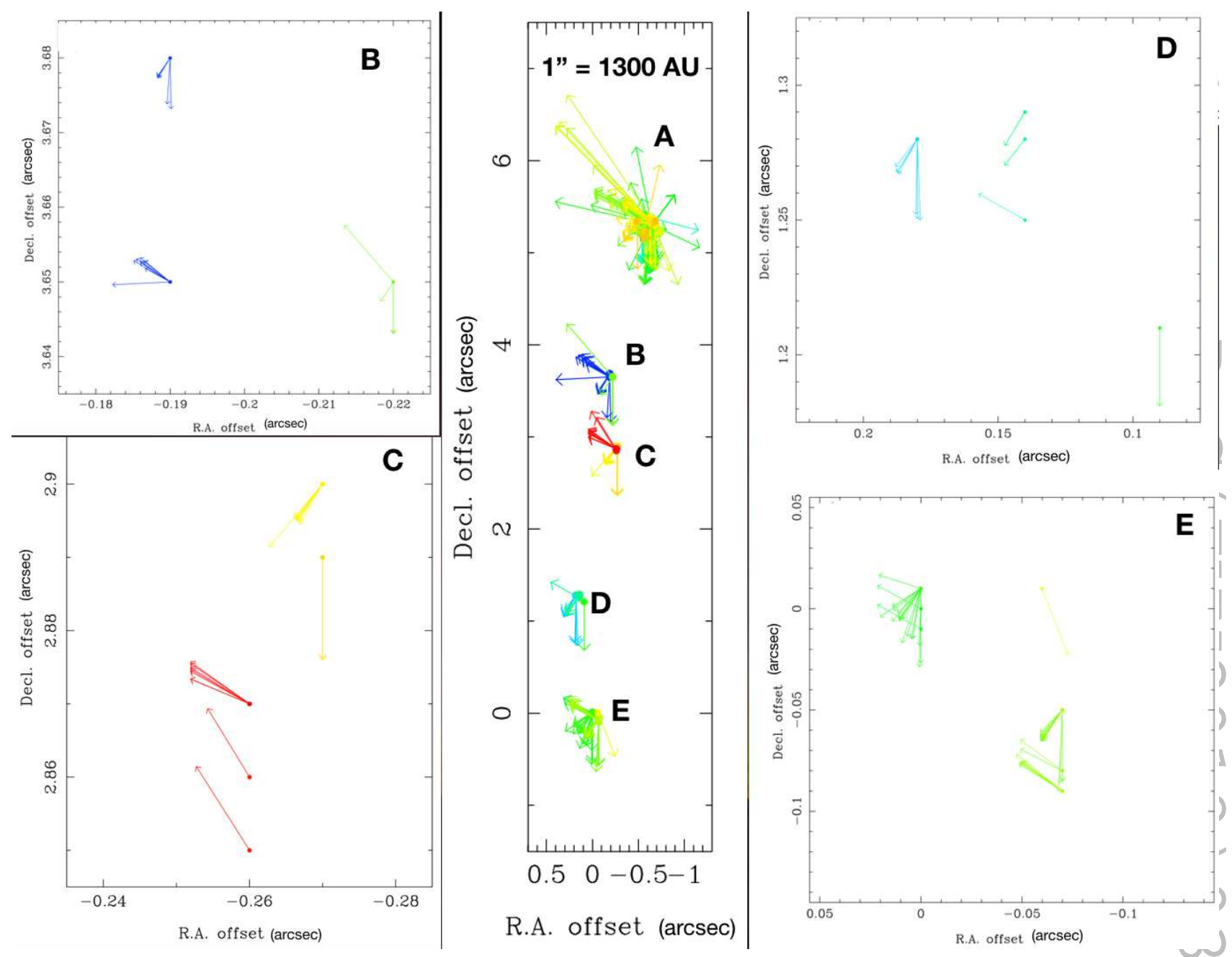

Figure 2: Relative proper motion of the maser features in clusters A - E traced from our 3-epoch observations.

The kinematics of the masers in cluster A is dominant (see Figure 3). It shows a turbulent flow of gas (rebound flow) around the bow-shock structure instead of an outward flow in the direction of the bipolar outflow.

\section{Discussions}

Our 3-epoch observations covered the peak of the $\mathrm{H}_{2} \mathrm{O}$ maser outburst and interferometric observations with JVLA confirmed the bursting masers to be located in CM2 object corresponding to the cluster A of out map (Brogan et al. 2018). We were able to capture the gas motion at the shocked interface that resulted in the enhancement of the flux densities of the masers.

As noted in section 3.2, we noticed a reverse flow of gas traced by our maser relative proper motion in cluster A, after a collision with shock interface, and thus must have led to the flaring of the masers. 


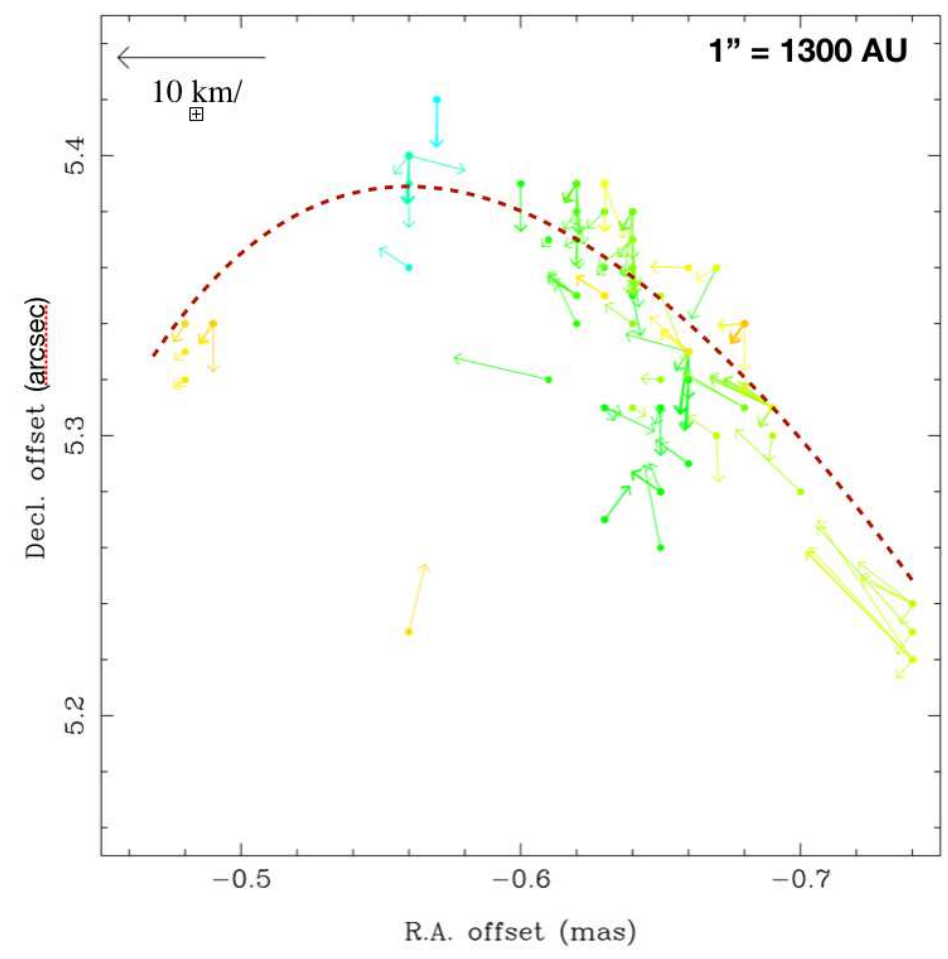

Figure 3: Zoom of the maser relative proper motion around cluster A.

These gas motion is driven by a young stellar object in MM1 (see Brogan et al. 2018; Hunter et al. 2017, 2018). There clear evidence of multiplicity of protostars in MM1 as suggested by the presence of multiple cores and multiple outflows from the region. High resolution observations will be required to unambiguously identify the sources driving the various outflows. We have continued other follow up observations of this target to understand more about the post-flare phase and the driving sources of the multiple outflow.

\section{References}

[1] Abraham, Z., Cohen, N. L., Opher, R., Raffaelli, J. C., \& Zisk, S. H. 1981, A\&A, 100, L10

[2] Brogan, C. L., Hunter, T. R., Cyganowski, C. J., et al. 2016, ApJ, 832, 187

[3] Brogan, C. L., Hunter, T. R., Cyganowski, C. J., et al. 2018, ApJ, 866, 87

[4] Chibueze, J. O., Omodaka, T., Handa, T., et al. 2014, ApJ, 784, 114

[5] Hirota, T., Tsuboi, M., Kurono, Y., et al. 2014, PASJ, 66, 106

[6] Honma, M., Yoon, K. C., Bushimata, T., et al. 2004, PASJ, 56, L15

[7] Hunter, T. R., Brogan, C. L., Megeath, S. T., et al. 2006, ApJ, 649, 888

[8] Hunter, T. R., Brogan, C. L., MacLeod, G., et al. 2017, ApJL, 837, L29

[9] Hunter, T. R., Brogan, C. L., MacLeod, G. C., et al. 2018, ApJ, 854, 170 
[10] Lekht, E. E., Pashchenko, M. I., Rudnitskii, G. M., \& Tolmachev, A. M. 2018, Astronomy Reports, 62,213

[11] Liljeström, T., \& Gwinn, C. R. 2000, ApJ, 534, 781

[12] MacLeod, G. C., Smits, D. P., Goedhart, S., et al. 2018, MNRAS, 478, 1077

[13] Omodaka, T., Maeda, T., Miyoshi, M., et al. 1999, PASJ, 51, 333

[14] Volvach, A. E., Volvach, L. N., MacLeod, G., et al. 2017, The Astronomer's Telegram, 10853, 